CLINICAL STUDY

\title{
Glycosylation of sera thyroglobulin antibody in patients with thyroid diseases
}

\author{
Lanlan Zhao, Mingming Liu, Ying Gao, Youyuan Huang, Guizhi Lu, Yanming Gao, Xiaohui Guo and Bingyin Shi ${ }^{1}$ \\ Department of Endocrinology, Peking University First Hospital, Beijing 100034, People's Republic of China and ${ }^{1}$ Department of Endocrinology, The First \\ Affiliated Hospital of Xi'an Jiaotong University School of Medicine, Xi'an, Shaanxi 710061, People's Republic of China
}

(Correspondence should be addressed to Y Gao; Email: bjgaoying@yahoo.com)

\begin{abstract}
Objective: Thyroglobulin antibody $(\mathrm{TgAb})$ is an important autoantibody in thyroid diseases, which is a glycoprotein, predominantly of IgG class. Glycosylation of the IgG-Fc contributes to many effector functions exhibited by antibodies. The aim of our study was to investigate the glycosylation of sera $\mathrm{TgAb}$ in patients with different thyroid diseases.

Design and methods: Sera from 146 patients were collected and divided into four groups: Hashimoto's thyroiditis (HT, $n=90$ ), Graves' disease (GD, $n=20$ ), papillary thyroid carcinoma (PTC, $n=17$ ), and PTC with histological lymphocytic thyroiditis (PTC-T, $n=19$ ). HT patients were further divided into euthyroidism and subclinical and overt hypothyroidism groups. Lectin-ELISAs were performed to detect the relative amount of core fucose, terminal galactose, and sialic acid on each $\mathrm{TgAb}$ respectively. Results: Among HT, GD, and PTC groups, HT patients had significantly lower core fucose content on $\mathrm{TgAb}$ than the other two groups; an increasing trend of sialylation was found in PTC sera $(P=0.076)$ compared with HT groups. PTC-T patients had significantly higher sialylated TgAb than HT and GD patients, and no significant difference was found between PTC and PTC-T. There was no significant difference in the three carbohydrate residue contents on sera $\mathrm{TgAb}$ among HT subgroups. In all the patients, negative correlation was found between sialic acid content and $\operatorname{TgAb} \operatorname{IgG}$ levels $(r=-0.736$, $P<0.001)$.

Conclusions: Our study showed that glycosylation of sera $\mathrm{TgAb}$ varied in different thyroid diseases and it might be involved in pathogenesis of thyroid disorders.
\end{abstract}

European Journal of Endocrinology 168 585-592

\section{Introduction}

Circulating thyroglobulin antibody $(\mathrm{TgAb})$ is a hallmark of autoimmune thyroid disease (AITD). It could be detected in 80-90\% patients with Hashimoto's thyroiditis (HT) and 50-70\% patients with Graves' disease (GD) (1). Compared with the general population, the prevalence of $\mathrm{TgAb}$ is increased nearly threefold in patients with differentiated thyroid carcinoma (2). $\mathrm{TgAb}$ can mediate antibody-dependent cell-mediated cytotoxicity (ADCC) in artificial systems (3) and it may be involved in thyroid destruction in AITD. In papillary thyroid carcinoma (PTC), as the presence of $\mathrm{TgAb}$ usually invalidates the serum $\mathrm{Tg}$ result, investigators have found that $\mathrm{TgAb}$ might be used as a surrogate tumor marker (4).

$\mathrm{TgAb}$ is predominantly of $\operatorname{IgG}$ class (5), and $\operatorname{IgG}$ is a glycoprotein with a sugar moiety attached to each of the asparagine 297 residues in the $\mathrm{CH} 2$-domains of the two Fc-fragments (6). Variable attachment of outer arm sugars (sialic acid, galactose, and fucose) to a heptasaccharide GlcNAc2Man3GlcNAc2 core structure results in the generation of heterogeneous array of $\operatorname{IgG}$ glycoforms. These glycoforms can differ in their efficacy of effector function activation as it influences binding of IgG molecules to $\mathrm{Fc}$ receptors and $\mathrm{Clq}(7)$.

As is known to all, glycosylation of IgG plays an important role in disease pathogenesis, not only in autoimmune disorders but also in tumors. It has been known that the absence of core fucose residues in the Fc glycans substantially increases the ADCC activity of IgG $(8,9)$. Increased sialylation of Fc glycans results in decreased ADCC activity $(10,11)$. Reduced IgG-Fc galactosylation has been documented to be limited mainly to patients with certain inflammatory and autoimmune diseases, such as rheumatoid arthritis (RA) and Crohn's disease (12, 13). Interestingly, higher levels of agalactosylated IgG oligosaccharides have recently been reported in patients with prostate cancer (14), ovarian cancer (15), and gastric cancer (16).

As far as we know, most studies on antibody glycosylation focused on total IgG; by contrast, there was little information on the glycosylation of a specific antibody. As AITD is an organ-specific disease, the study on $\mathrm{TgAb}$ glycosylation might provide more information on humoral factors in the pathogenesis of thyroid 
diseases. The aim of our study was to investigate the glycosylation of sera $\mathrm{TgAb}$ in patients with different thyroid diseases including HT, GD, PTC, and PTC with histological lymphocytic thyroiditis (PTC-T).

\section{Materials and methods}

\section{Study groups}

A total of 146 subjects (137 women and nine men) with thyroid disorders were consecutively referred to Peking University First Hospital in the period December 2010 to August 2012 and enrolled in the current study before receiving treatment. The study groups included 110 AITD (including 90 HT and 20 GD), 17 PTC (without HT or GD), and 19 PTC-T patients confirmed by histopathology. All of them had $\mathrm{TgAb}$, which were detected by electrochemiluminescence immunoassays. The PTC-T group included $57.9 \%$ of patients with a clinical diagnosis of nodular thyroid disease (NTD) without any clinical features of HT. None of the PTC or PTC-T patients had been previously treated with ${ }^{131} \mathrm{I}$. None of the patients had evidence of hereditary or acquired variations in the concentration of thyroxinebinding globulin. There was no evidence of other autoimmune diseases, including systemic lupus erythematosus, RA, type 1 diabetes mellitus, or pernicious anemia. The HT patients were further divided into three subgroups according to thyroid function: patients with euthyroidism $(\mathrm{Eu}, n=30)$, subclinical hypothyroidism $(\mathrm{sH}, n=26)$, and overt hypothyroidism $(\mathrm{H}, n=34)$. This study complied with the Helsinki declaration and was approved by the Ethics Committee of Peking University First Hospital. All the patients gave written informed consent.

\section{Detection of thyroid function and $\mathrm{TgAb}$}

Sera samples of all the patients were collected at diagnosis and kept frozen at $-80{ }^{\circ} \mathrm{C}$ until use. Chemiluminescence immunoassays were used to detect total triiodothyronine $\left(\mathrm{TT}_{3}\right)$, total tetraiodothyronine $\left(\mathrm{TT}_{4}\right)$, and TSH (ADVIA Centaur (Siemens Healthcare Diagnostics, Tarrytown, NY, USA)). TgAb was detected by electrochemiluminescence immunoassays (Cobas e 601 Analyzer (Roche Diagnostics)).

\section{Detection of total TgAb IgG by antigen-specific ELISAs}

Half of the 96-well microtiter plates (Costar, Data Packaging Corporation, Spencer, MA, USA) were coated with $4 \mu \mathrm{g} / \mathrm{ml}$ human native $\mathrm{Tg}$ (Calbiochem Merck $\mathrm{KGaA}$ ) in $0.05 \mathrm{~mol} / \mathrm{l}$ bicarbonate buffer ( $\mathrm{pH}$ 9.6) for $90 \mathrm{~min}$ and the other half were coated with bicarbonate buffer alone to act as antigen-free wells. The volume in each well was $50 \mu \mathrm{l}$ in all the steps. All incubations were carried out at $37^{\circ} \mathrm{C}$ for $1 \mathrm{~h}$ unless otherwise specified, and the plates were washed three times with PBS containing $0.1 \%$ Tween 20 (PBST) between stages. After washing, all the plates were blocked with 3\% BSA (Sigma) and were then oxidized with $200 \mu \mathrm{l} 0.1 \mathrm{~mol} / \mathrm{l}$ sodium periodate (17) in citrate buffer $(\mathrm{pH} 4.0)$ for $10 \mathrm{~min}$ at room temperature in the dark. The serum samples diluted 1:100 were added in duplicate to both antigen-coated and antigen-free wells. Every plate contained a positive control, a negative control, and a blank. Then, an HRP conjugate goat anti-human immunoglobulin (Jackson ImmunoResearch Laboratories, Inc., Baltimore Pike, West Grove, PA, USA), 1:2500 dilution, was subsequently employed for antibody detection. Following washing, ortho-phenylenediamine diluted in citrate buffer containing $0.1 \%$ hydrogen peroxide was used as substrate/chromogen mixture. The reaction was stopped by the addition of $1 \mathrm{~mol} / \mathrm{l}$ hydrochloric acid. Finally, the results were recorded at $490 \mathrm{~nm}$ by a Varioskan Flash Multimode Reader (Thermo Fisher Scientific, Inc., Waltham, MA, USA) and expressed as percentages of the positive control.

\section{Detection of carbohydrate residues on sera TgAb by lectin-ELISAs}

Lectin-ELISAs (L-ELISA) were applied to detect carbohydrate on sera TgAb. Three biotinylated lectins (Vector Laboratories, Burlingame, CA, USA) were used in the current study: Aleuria Aurantia Lectin (AAL) (18) for fucose detection, Ricinus Communis Agglutinin I (RCA I) (19) for galactose detection, and Elderberry lectin (EBL) (20) for sialic acid detection. Similar assay procedures as mentioned earlier were conducted to detect sera $\mathrm{TgAb}$ glycosylation. Briefly, after the sera diluted 1:100 were added in duplicate to both antigencoated and antigen-free wells, diluted biotinylated AAL (1:1000), RCA I (1:500), and EBL (1:2000) were employed respectively and then peroxidase-labeled avidin D (Vector Laboratories) (1:10 000 for AAL and 1:12 000 for RCA I and EBL) was added. The subsequent procedures were the same as described earlier. The results were expressed as percentages of the positive control as well. The relative amount of fucose in each $\mathrm{TgAb}$ IgG was calculated as the percentage of fucose-positive control/the percentage of TgAb IgG-positive control $\times 100 \%$. The calculation of the relative amount of galactose and sialic acid was performed similarly.

\section{Statistical analysis}

Statistical analysis was performed using the SPSS 13.0 (SPSS) statistics package. Comparisons were carried out by the Mann-Whitney $U$ test, one-way ANOVA, $\chi^{2}$ test, Kruskal-Wallis $H$ test, Pearson correlation, and Spearman's test. A $P$ value under 0.05 was considered statistically significant. 
Table 1 Demographic data, thyroid functional status, and TgAb IgG levels of the patients in different groups. Numbers expressed as mean \pm s.D., median (interquartile range). Reference value range: $\mathrm{TT}_{3}: 0.92-2.79 \mathrm{nmol} / \mathrm{l} ; \mathrm{TT}_{4}: 58.1-140.6 \mathrm{nmol} / \mathrm{l} ; \mathrm{TSH}: 0.35-5.5 \mathrm{mIU} / \mathrm{l}$.

\begin{tabular}{lllll}
\hline Groups & HT $(n=90)$ & GD $(n=20)$ & PTC $(n=17)$ & PTC-T $(n=19)$ \\
\hline Age (years) & $50.5(33.5-57.0)^{*}$ & $34.0(23.0-41.7)$ & $54.0(22.4-64.0)^{*}$ & $48.0(41.8-52.0)^{*}$ \\
Gender (M/F) & $6 / 84$ & $0 / 20$ & $2 / 15$ & $1 / 18$ \\
$\mathrm{TT}_{3}(\mathrm{nmol} / \mathrm{l})$ & $1.5(1.3-1.8)^{*}$ & $4.6(3.6-8.5)$ & $1.6(1.4-1.8)^{*}$ & $1.6(1.4-1.9)^{*}$ \\
$\mathrm{TT}_{4}(\mathrm{nmol} / \mathrm{l})$ & $88.6(64.7-104.1)^{*,+, \neq}$ & $201.9(133.1-249.1)$ & $112.0(89.6-122.6)^{\star}$ & $113.4(100.1-127.9)^{*}$ \\
$\mathrm{TSH}_{(\mathrm{mlU} / \mathrm{l})}$ & $6.4(4.3-18.0)^{*,+,}$ & $0.01(0.00-0.04)$ & $2.3(1.3-4.1)^{*}$ & $2.5(1.8-3.4)^{*}$ \\
Positive percentage & $41.2(33.2-62.4)$ & $48.6(39.6-61.8)$ & $46.3(38.6-52.8)$ & $39.2(32.7-55.1)$ \\
$\quad$ of TgAb IgG (\%) & & & & \\
\hline
\end{tabular}

HT, Hashimoto's thyroiditis; GD, Graves' disease; PTC, papillary thyroid carcinoma; PTC-T, papillary thyroid carcinoma with histological lymphocytic thyroiditis. ${ }^{\star} P<0.05$ vs GD; ${ }^{\dagger} P<0.05$ vs PTC; ${ }^{\ddagger} P<0.05$ vs $\mathrm{PTC}-\mathrm{T}$.

\section{Results}

\section{Demographic data of all the patients}

As shown in Table 1, there was no significant difference in gender distribution in HT, GD, PTC, and PTC-T groups. GD patients were younger than the other three groups $\left(P<0.05\right.$ respectively). $\mathrm{TT}_{3}$ and $\mathrm{TT}_{4}$ levels in GD group were significantly higher than those in the other three groups $\left(P<0.05\right.$ respectively). $\mathrm{TT}_{4}$ in the HT group was lower than that in PTC and PTC-T groups $(P<0.05)$. TSH levels in HT were significantly higher than those in the other three groups $(P<0.05$ respectively), and no significant difference was found in TSH levels between PTC and PTC-T groups.

In HT patients, there were no significant differences in age and gender distribution in patients of $\mathrm{H}, \mathrm{sH}$, and $\mathrm{Eu}$ subgroups. $\mathrm{TT}_{3}$ and $\mathrm{TT}_{4}$ levels in $\mathrm{H}$ were significantly lower than those in the other two groups $(P<0.05$ respectively) (Table 2), and TSH levels in the $\mathrm{H}$ group was significantly higher than those in the $\mathrm{sH}$ and $\mathrm{Eu}$ groups $(P<0.05$ respectively $)$.

\section{Oxidation of thyroglobulin antigen}

As $\mathrm{Tg}$ is a glycoprotein, sodium periodate was used to cleave $\mathrm{Tg}$ carbohydrate in order to minimize the interference in the detection of glycosylation on sera TgAb. As shown in Fig. 1, all three carbohydrate residues on $\mathrm{Tg}$ could be cleft by sodium periodate, and terminal sialic acid was the most susceptible for the oxidation cleavage. As shown in Fig. 2, with prolongation of oxidation reaction, the OD values of $\mathrm{TgAb}$ binding oxidized $\mathrm{Tg}$ fell only slightly, which indicated that $\mathrm{Tg}$ oxidation might have little influence on its antigenicity. Ten minutes was finally chosen as the reaction conditions with sodium periodate, giving consideration to the detection of $\operatorname{TgAb} \operatorname{IgG}$ and all the three carbohydrate residues.

\section{Results of total TgAb IgG levels}

As shown in Table 1, there was no significant difference in sera TgAb IgG levels among the four groups. In HT patients (Table 2), TgAb IgG levels in the Eu group were significantly lower than those in the H group $(P<0.05)$, and there was no significant difference between the $\mathrm{sH}$ and $\mathrm{H}$ groups $(P>0.05)$.

\section{Results of carbohydrate residues on each TgAb}

The relative amount of three carbohydrate residues on each TgAb in HT, GD, PTC, and PTC-T groups is shown in Fig. 3 respectively.

The relative amount of fucose on sera TgAb in different thyroid diseases Among HT, GD, and PTC groups, sera TgAb in HT patients had significantly lower core fucose content than that in the other two groups $(P<0.05$ respectively). No significant difference was found between GD and PTC groups and PTC and PTC-T groups. There was significantly less fucosylated TgAb in HT sera than in PTC-T groups $(P=0.001)$.

Table 2 Demographic data, thyroid functional status, and TgAb IgG levels of the patients in the HT subgroups. Numbers expressed as mean \pm s.D., median (interquartile range). Reference value range: $\mathrm{TT}_{3}$ : 0.92-2.79 nmol//; TT 4 : 58.1-140.6 nmol//; TSH: 0.35-5.5 mlU/l.

\begin{tabular}{llll}
\hline Groups & Eu $(n=30)$ & sH $(n=26)$ & H $(n=34)$ \\
\hline Age (years) & $44.7 \pm 16.0$ & $50.0 \pm 12.5$ & $47.0 \pm 14.0$ \\
Gender (M/F) & $1 / 29$ & $1 / 25$ & $4 / 30$ \\
$\mathrm{TT}_{3}(\mathrm{nmol} / \mathrm{l})$ & $1.6(1.4-1.7)^{\dagger}$ & $1.8(1.6-2.0)^{\dagger}$ & $1.2(1.0-1.5)$ \\
$\mathrm{TT}_{4}(\mathrm{nmol} / \mathrm{l})$ & $98.1(85.0-107.0)^{\dagger}$ & $94.5(85.5-108.0)^{\dagger}$ & $53.0(37.2-71.0)$ \\
$\mathrm{TSH}^{\dagger}(\mathrm{mlU} / \mathrm{l})$ & $3.4(2.0-4.5)^{*, \dagger}$ & $6.5(6.1-8.4)^{\dagger}$ & $39.6(10.2-100.3)$ \\
Positive percentage & $35.7(31.3-47.8)^{\dagger}$ & $46.8(34.4-64.1)$ & $52.7(33.8-67.0)$ \\
$\quad$ & & & \\
\hline
\end{tabular}

Eu, euthyroidism; sH, subclinical hypothyroidism; $\mathrm{H}$, hypothyroidism. ${ }^{*} P<0.05 \mathrm{vs} \mathrm{sH} ;{ }^{\dagger} P<0.05$ vs $\mathrm{H}$. 

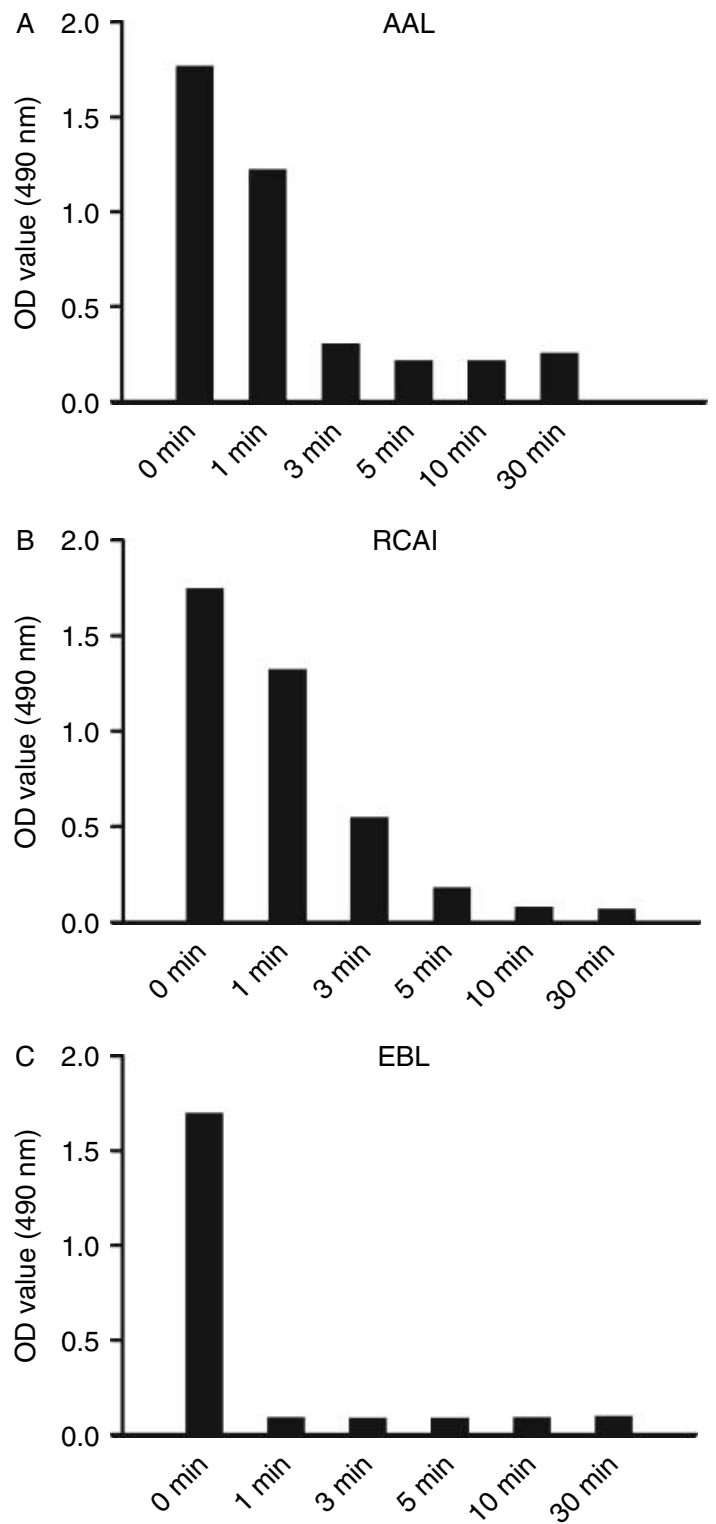

Figure 1 The biotinylated lectins binding to thyroglobulin $(\mathrm{Tg})$ in different oxidation time with sodium periodate. (A) Aleuria Aurantia lectin (AAL) for fucose detection, (B) Ricinus Communis Agglutinin I (RCA I) for galactose detection, and (C) Elderberry lectin (EBL) for sialic acid detection.

The galactose on sera $\mathrm{TgAb}$ in different thyroid diseases No significant differences in $\mathrm{TgAb}$ galactosylation were found among the four groups.

The relative amount of sialic acid on sera $\mathrm{TgAb}$ in different thyroid diseases Although there was no significant difference in the relative amount of sialic acid on each TgAb in the HT, GD, and PTC groups, an increasing trend of sialylation was found in PTC sera compared with the HT group $(P=0.076)$. PTC-T patients had significantly more sialylated $\mathrm{Tg} A b$ than HT and GD patients $(P=0.005$ and $P=0.021$ respectively).
The three carbohydrate residues on $\mathrm{TgAb}$ in $\mathrm{HT}$ subgroups There were no significant differences in the relative amount of all the three carbohydrate residues on $\mathrm{TgAb}$ among the three HT subgroups (data not shown).

The relationship between carbohydrate residues on TgAb and TgAb IgG levels For all the patients with different thyroid diseases, a negative relationship was found between the relative amount of sialic acid and TgAb IgG levels $(r=-0.736, P<0.001)$ (Fig. 4). There were no relationships between the levels of glycosylation for all three carbohydrate residues and the levels of TSH, $\mathrm{TT}_{3}$, and $\mathrm{TT}_{4}$ respectively.

\section{Discussion}

During the last decade, it became apparent that glycosylation of the IgG-Fc, the most important step of posttranslational modification, contributes to many effector functions exhibited by antibodies such as complement binding and activation, induction of ADCC, and binding to macrophage Fc receptors (21). Notable alterations in glycosylation of the IgG-Fc region have been described not only in autoimmune disorders but also in some malignancy diseases (16). For example, Chen et al. (22) had reported that the changes in total IgG-Fc $N$-linked glycosylation was associated with thyroid cancer.

Structural features of glycan moieties in the $\mathrm{Fc}$ portion of $\operatorname{IgG}$ were regulated in an antigen-specific fashion (23). As $\operatorname{TgAb}$ is produced by thyroid-derived lymphocytes, and B cells at the site of chronic inflammation might be the major source of differentially glycosylated (auto)antibodies (24), we studied the glycosylation in thyroid-specific antibody rather than total IgG in different thyroid diseases.

In our study, the levels of fucosylation and sialylation on $\operatorname{TgAb}$ varied in different thyroid disorders. The IgG

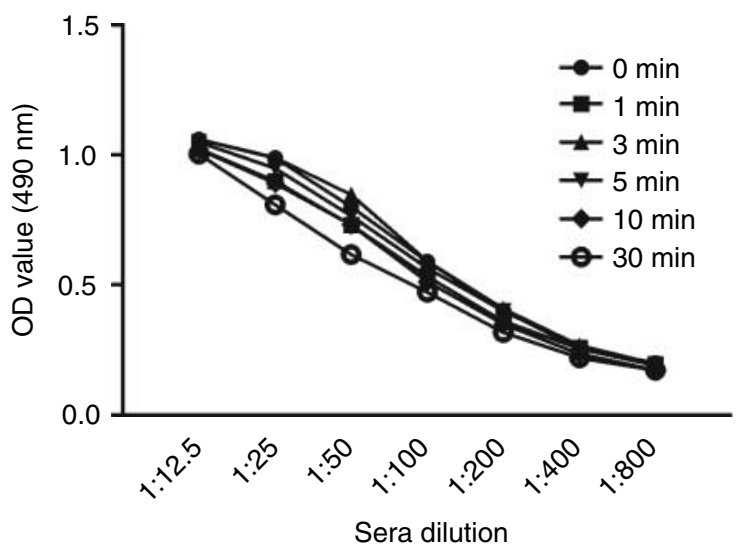

Figure $2 \mathrm{TgAb} \operatorname{lgG}$ binding to thyroglobulin with a serial dilution (diluted 1:12.5-1:800) in different oxidation times with sodium periodate. 
A
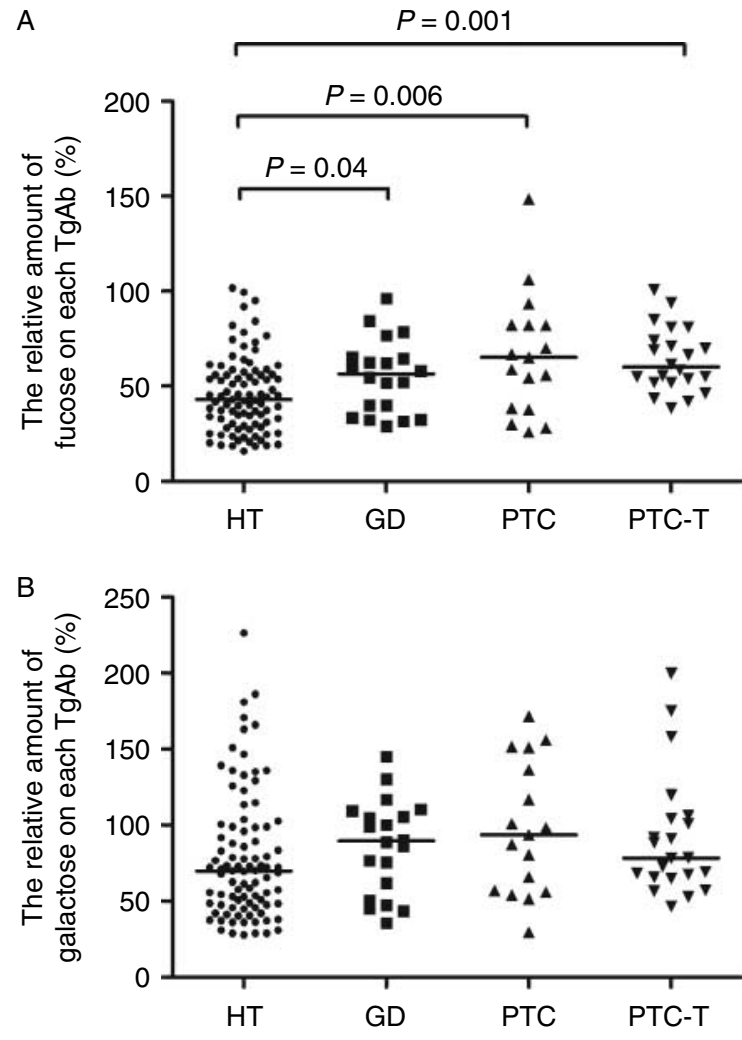

C
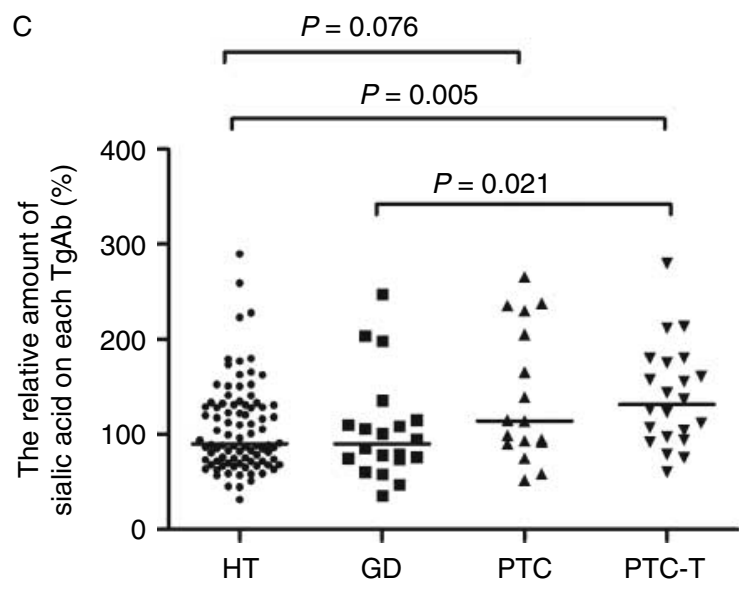

Figure 3 Comparisons of the relative amount of carbohydrate residues on each sera $\mathrm{TgAb}$ from patients with different thyroid diseases. (A) Sera TgAb in HT patients had significantly lower core fucose content than that in the GD, PTC, and PTC-T groups. (B) There was no significant difference in the relative galactosylation on $\mathrm{TgAb}$ in different thyroid groups. (C) The relative amount of sialic acid on each sera TgAb from HT and GD patients was lower than that from patients with PTC-T. HT, Hashimoto's thyroiditis $(n=90)$; GD, Graves' disease $(n=20)$; PTC, papillary thyroid carcinoma $(n=17)$; PTC-T, papillary thyroid carcinoma with histological lymphocytic thyroiditis $(n=19)$. molecule comes in close contact if fucose residues are present (25); therefore, non-fucosylated antibodies bind to the Fc $\gamma$ RIIIa receptor with significantly increased affinity. By this way, the commercial therapeutic monoclonal antibody such as trastuzumab (26) and rituximab (27) is constantly improved to work more efficiently. It has also been reported that the sialic acidcontaining $\operatorname{IgG}$ displays an anti-inflammatory effect (11) and sialic acid negatively affects antibody binding to the FcrRIIIa. In the current study, among the HT, GD, and PTC groups, the lowest fucosylation levels of TgAb were found in HT sera, and an increasing trend in sialylated $\mathrm{TgAb}$ was found in the PTC group compared with the HT group. As non-fucosylated and nonsialylated antibodies may have stronger ability to participate in ADCC, we speculated that low levels of fucosylation and sialylation might be the property of $\mathrm{TgAb}$ in HT, which might have more capacity to induce thyroid destruction.

Current evidence does not support an important role of terminal galactose residues in either enhancing or attenuating the activity of IgG in vivo (6). And in some reports, terminal galactose content of IgG does not affect ADCC but complement-dependent cytotoxicity activity (28). In our study, no significant difference was found in the galactosylation on $\mathrm{TgAb}$ in the AITD, PTC, and PTC-T groups. As TgAb does not fix the complement (29), the importance of galactose residues on $\mathrm{TgAb}$ need to be further studied.

In thyroid cancer, the $\mathrm{TgAb}$ concentration can serve as a surrogate tumor marker for recurrence (4). Whether different pathogenesis in AITD and PTC is involved in the production of $\mathrm{TgAb}$ is still not known. Latrofa et al. (30) found different epitope specificities for $\mathrm{TgAb}$ in PTC compared with AITD. Our study found that the levels of $\mathrm{TgAb}$ glycosylation were also different between AITD and PTC groups. As different B-cell subsets produce differentially glycosylated $\operatorname{IgG}(31)$, we assumed that $\mathrm{TgAb}$ arose in PTC from different pathogenetic mechanisms of AITD. In PTC, TgAb production might be the result of an immune response to the inflammation associated with tumorigenesis that may have the potential to release posttranslational modified $\mathrm{Tg}$ antigens with enhanced immunogenicity (4).

In the current study, the PTC-T group, confirmed by histopathology, included $57.9 \%$ of patients with a clinical diagnosis of NTD. Latrofa et al. (30) had found that $\mathrm{TgAb}$ epitope pattern in PTC-T resembled that of AITD. As only a few PTC-T patients had classical clinical features of HT in our research, no significant difference in the levels of $\operatorname{TgAb}$ glycosylation was found between PTC and PTC-T groups.

It was interesting that the levels of sialylation on each $\mathrm{TgAb}$ had a negative relationship with $\mathrm{TgAb}$ IgG levels, independent of thyroid function. In an epidemiological study, the individuals with thyroid antibodies might be at high risk of developing thyroid failure (32). We speculated that the changing of carbohydrate residues 
on each $\operatorname{Tg} A b \operatorname{IgG}$ might be a consequence of elevated Ig synthesis by B cells (33). Chen et al. (34) had reported that breaking B-cell self-tolerance occurred first for $\mathrm{Tg}$ and subsequently for TPO, and $\mathrm{TgAb}$ arose first followed later by TPOAb in HT. We assumed that with $\mathrm{TgAb}$ levels increasing, more $\mathrm{TgAbs}$ with lower terminal sialic acid were produced, which might have higher capacity in mediating ADCC and contributing to thyrocyte damage, and this process triggered thyroid autoimmunity further.
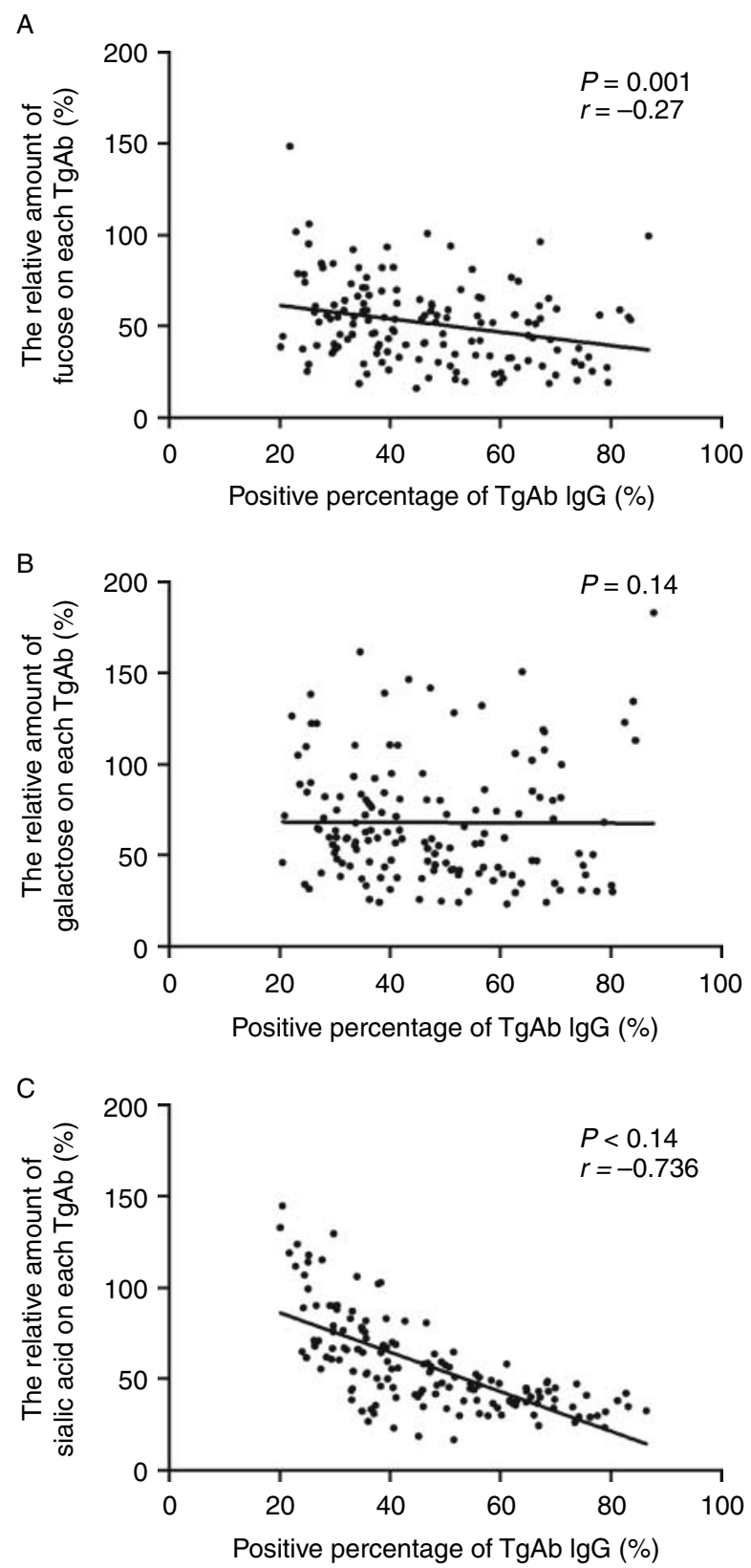

Figure 4 The correlation between the relative amount of carbohydrate residues on each $\mathrm{TgAb}$ and $\mathrm{TgAb}$ IgG levels in all the patients $(n=146)$. There was a negative correlation between the relative amount of sialic acid and TgAb IgG levels. (A) Core fucose, (B) galactose residue, and (C) sialic acid residue.
Overall, as sugar residues such as fucose and sialic acid can dramatically alter IgG activity, the change in IgG glycosylation on thyroid antibodies might provide a new view to investigate the role of autoantibodies in the pathogenesis of thyroid diseases.

In clinical practice, HT patients with $\mathrm{TgAb}$ may have different thyroid functional status. It is an evolutionary process from Eu to sH, and even to H (35). When thyroid cell hyperplasia cannot compensate for follicular destruction and $\sim 90 \%$ of the thyroid gland is destroyed, thyroid failure will develop (36). As there were no significant differences in the levels of glycosylation on each $\operatorname{TgAb}$ among the three HT subgroups with different thyroid functional status, the levels of glycosylation on $\mathrm{TgAb}$ might not represent thyrocyte hyperplasia but merely reflect the capacity of inducing thyroid destruction by ADCC.

In the current study, the total glycosylation of $\mathrm{TgAb}$ was measured by antigen-specific ELISAs. TgAb is predominantly of IgG class, and IgA and IgM isotype could be detected only in sera from a few patients and at much lower levels (5). Therefore, compared with the $\mathrm{N}$-linked glycosylation of $\mathrm{TgAb}$ IgG, glycosylation of $\operatorname{IgA}$ and IgM (37) might be neglected. And it is well known that human serum IgG has on average $2.8 \mathrm{~N}$-glycosidetype sugar chains per protein molecule. Two of them are invariably located in the conserved $\mathrm{N}$-glycosylation site of asparagine 297, and additional ones are found in the variable regions of the light and heavy chains (38). In our study, we did not distinguish the glycosylation on $\mathrm{TgAb}$ between IgG-Fab and Fc fragments. Further study focused on Fc regions might provide more convincing evidence on the role of the glycosylation on $\mathrm{TgAb}$ in the pathogenesis of thyroid diseases.

In consideration of the sample size and the cost for detection, the simple L-ELISAs were used to detect relative glycosylation on $\mathrm{TgAb}$ in our study, instead of more complex but more accurate assays such as liquid chromatography mass spectrometry and high performance liquid chromatography. Furthermore, removal of the terminal sugar residues from $\mathrm{Tg}$ by oxidation is a necessary step to avoid the antigen's reactivity with the lectins used in this study. Sodium periodate is a strong oxidant and can cleave the carbohydrate nonspecifically. After the oxidation step, the ability of $\mathrm{TgAb}$ IgG binding to oxidized $\mathrm{Tg}$ changed little. This result was in accord with the notion that the $\mathrm{TgAb}$ epitope does not involve carbohydrate (39). In addition, an oxidation time of 10 min was used in ELISAs for TgAb IgG detection and L-ELISAs in order to guarantee the same amount of $\mathrm{TgAb}$ IgG was captured in these assays.

Taken together, our study showed for the first time that glycosylation of sera TgAb varied in AITD and PTC, and that the levels of sialylation of $\mathrm{TgAb}$ might decrease with increasing $\operatorname{TgAb}$ levels. Further studies on the glycosylation of thyroid autoantibodies may be helpful to elucidate the potential role of autoantibodies in the pathogenesis of thyroid diseases. 


\section{Declaration of interest}

The authors declare that there is no conflict of interest that could be perceived as prejudicing the impartiality of the research reported.

\section{Funding}

This work was supported by Beijing Natural Science Foundation (grant numbers 7122185), Beijing Nova Program (grant numbers 2011011), and Program for New Century Excellent Talents in University (grant numbers BMU20110266) and Sector Funds of Ministry of Health (grant numbers 201002002).

\section{References}

1 Hollowell JG, Staehling NW, Flanders WD, Hannon WH, Gunter EW, Spencer CA \& Braverman LE. Serum TSH, T(4), and thyroid antibodies in the United States population (1988 to 1994): National Health and Nutrition Examination Survey (NHANES III). Journal of Clinical Endocrinology and Metabolism 200287 489-499. (doi:10.1210/jc.87.2.489)

2 Spencer CA, Takeuchi M, Kazarosyan M, Wang CC, Guttler RB, Singer PA, Fatemi S, LoPresti JS \& Nicoloff JT. Serum thyroglobulin autoantibodies: prevalence, influence on serum thyroglobulin measurement, and prognostic significance in patients with differentiated thyroid carcinoma. Journal of Clinical Endocrinology and Metabolism $1998 \mathbf{8 3}$ 1121-1127. (doi:10.1210/ jc.83.4.1121)

3 Calder EA, Penhale WJ, McLeman D, Barnes EW \& Irvine WJ. Lymphocyte-dependent antibody-mediated cytotoxicity in Hashimoto thyroiditis. Clinical and Experimental Immunology 197314 $153-158$.

4 Spencer CA. Clinical review: clinical utility of thyroglobulin antibody $(\mathrm{TgAb})$ measurements for patients with differentiated thyroid cancers (DTC). Journal of Clinical Endocrinology and Metabolism 201196 3615-3627. (doi:10.1210/jc.2011-1740)

5 Torrigiani G, Roitt IM \& Doniach D. Ouantitative distribution of human thyroglobulin autoantibodies in different immunoglobulin classes. Clinical and Experimental Immunology 19683 621-630.

6 Lux A \& Nimmerjahn F. Impact of differential glycosylation on IgG activity. Advances in Experimental Medicine and Biology $2011 \mathbf{7 8 0}$ 113-124. (doi:10.1007/978-1-4419-5632-3_10)

7 Raju TS. Terminal sugars of Fc glycans influence antibody effector functions of IgGs. Current Opinion in Immunology 2008 20 471-478. (doi:10.1016/j.coi.2008.06.007)

8 Shields RL, Lai J, Keck R, O'Connell LY, Hong K, Meng YG, Weikert SH \& Presta LG. Lack of fucose on human IgG1 N-linked oligosaccharide improves binding to human Fcgamma RIII and antibody-dependent cellular toxicity. Journal of Biological Chemistry 2002277 26733-26740. (doi:10.1074/jbc.M202069200)

9 Satoh M, Iida S \& Shitara K. Non-fucosylated therapeutic antibodies as next-generation therapeutic antibodies. Expert Opinion on Biological Therapy 20066 1161-1173. (doi:10.1517/ 14712598.6.11.1161)

10 Scallon BJ, Tam SH, McCarthy SG, Cai AN \& Raju TS. Higher levels of sialylated Fc glycans in immunoglobulin G molecules can adversely impact functionality. Molecular Immunology $2007 \mathbf{4 4}$ 1524-1534. (doi:10.1016/j.molimm.2006.09.005)

11 Kaneko Y, Nimmerjahn F \& Ravetch JV. Anti-inflammatory activity of immunoglobulin $\mathrm{G}$ resulting from Fc sialylation. Science 2006313 670-673. (doi:10.1126/science.1129594)

12 Axford JS, Sumar N, Alavi A, Isenberg DA, Young A, Bodman KB \& Roitt IM. Changes in normal glycosylation mechanisms in autoimmune rheumatic disease. Journal of Clinical Investigation 199289 1021-1031. (doi:10.1172/JCI115643)

13 Cremata JA, Sorell L, Montesino R, Garcia R, Mata M, Cabrera G, Galvan JA, Garcia G, Valdes R \& Garrote JA. Hypogalactosylation of serum IgG in patients with coeliac disease. Clinical and Experimental Immunology 2003133 422-429. (doi:10.1046/ j.1365-2249.2003.02220.x)

14 Kanoh Y, Mashiko T, Danbara M, Takayama Y, Ohtani S, Egawa S, Baba S \& Akahoshi T. Changes in serum IgG oligosaccharide chains with prostate cancer progression. Anticancer Research 2004 24 3135-3139.

15 Gercel-Taylor C, Bazzett LB \& Taylor DD. Presence of aberrant tumor-reactive immunoglobulins in the circulation of patients with ovarian cancer. Gynecologic Oncology 200181 71-76. (doi:10.1006/gyno.2000.6102)

16 Kodar K, Stadlmann J, Klaamas K, Sergeyev B \& Kurtenkov O. Immunoglobulin G Fe N-glycan profiling in patients with gastric cancer by LC-ESI-MS: relation to tumor progression and survival. Glycoconjugate Journal 201229 57-66. (doi:10.1007/s10719011-9364-z)

17 Keusch J, Levy Y, Shoenfeld Y \& Youinou P. Analysis of different glycosylation states in IgG subclasses. Clinica Chimica Acta 1996 252 147-158. (doi:10.1016/0009-8981(96)06326-7)

18 Radziejewska I, Borzym-Kluczyk M, Kisiel DG, Namiot Z, Wosek J \& Gindzienski A. Characterisation of glycoforms of ascitic fluids in benign and malignant diseases. Clinical Biochemistry 200942 72-77. (doi:10.1016/j.clinbiochem.2008.09.114)

19 Pasek M, Duk M, Podbielska M, Sokolik R, Szechinski J, Lisowska E \& Krotkiewski H. Galactosylation of IgG from rheumatoid arthritis (RA) patients - changes during therapy. Glycoconjugate Journal 200623 463-471. (doi:10.1007/s10719-006-5409-0)

20 Mondal G, Chatterjee U, Chawla YK \& Chatterjee BP. Alterations of glycan branching and differential expression of sialic acid on alpha fetoprotein among hepatitis patients. Glycoconjugate Journal 2011 28 1-9. (doi:10.1007/s10719-010-9316-z)

21 Endo T, Wright A, Morrison SL \& Kobata A. Glycosylation of the variable region of immunoglobulin $\mathrm{G}$ - site specific maturation of the sugar chains. Molecular Immunology 199532 931-940. (doi:10.1016/0161-5890(95)00078-S)

22 Chen G, Wang Y, Oiu L, Oin X, Liu H, Wang X, Song G, Li F, Guo Y, Guo $S$ et al. Human IgG Fc-glycosylation profiling reveals associations with age, sex, female sex hormones and thyroid cancer. Journal of Proteomics 201275 2824-2834. (doi:10.1016/ j.jprot.2012.02.001)

23 Wang J, Balog CI, Stavenhagen K, Koeleman CA, Scherer HU, Selman MH, Deelder AM, Huizinga TW, Toes RE \& Wuhrer M. $\mathrm{Fc}$-glycosylation of IgG1 is modulated by B-cell stimuli. Molecular and Cellular Proteomics 201110 M110.004655. (doi:10.1074/ mcp.M110.004655)

24 Weetman AP, McGregor AM, Lazarus JH \& Hall R. Thyroid antibodies are produced by thyroid-derived lymphocytes. Clinical and Experimental Immunology 198248 196-200.

25 Ferrara C, Stuart F, Sondermann P, Brunker P \& Umana P. The carbohydrate at FcgammaRIIIa Asn-162. An element required for high affinity binding to non-fucosylated IgG glycoforms. Journal of Biological Chemistry 2006281 5032-5036. (doi:10.1074/jbc. M510171200)

26 Junttila TT, Parsons K, Olsson C, Lu Y, Xin Y, Theriault J, Crocker L, Pabonan O, Baginski T, Meng G et al. Superior in vivo efficacy of afucosylated trastuzumab in the treatment of HER2amplified breast cancer. Cancer Research 201070 4481-4489. (doi:10.1158/0008-5472.CAN-09-3704)

27 Mori K, Iida S, Yamane-Ohnuki N, Kanda Y, Kuni-Kamochi R, Nakano R, Imai-Nishiya H, Okazaki A, Shinkawa T, Natsume A et al. Non-fucosylated therapeutic antibodies: the next generation of therapeutic antibodies. Cytotechnology $2007 \quad 55$ 109-114. (doi:10.1007/s10616-007-9103-2)

28 Hodoniczky J, Zheng YZ \& James DC. Control of recombinant monoclonal antibody effector functions by Fc $N$-glycan remodeling in vitro. Biotechnology Progress 200521 1644-1652. (doi:10.1021/bp050228w)

29 Weetman AP. Autoimmune thyroid disease. Autoimmunity 2004 37 337-340. (doi:10.1080/08916930410001705394)

30 Latrofa F, Ricci D, Grasso L, Vitti P, Masserini L, Basolo F, Ugolini C, Mascia G, Lucacchini A \& Pinchera A. Characterization of 
thyroglobulin epitopes in patients with autoimmune and non-autoimmune thyroid diseases using recombinant human monoclonal thyroglobulin autoantibodies. Journal of Clinical Endocrinology and Metabolism 200893 591-596. (doi:10.1210/ jc.2007-1199)

31 Wuhrer M, Porcelijn L, Kapur R, Koeleman CA, Deelder A, de Haas M \& Vidarsson G. Regulated glycosylation patterns of IgG during alloimmune responses against human platelet antigens. Journal of Proteome Research 2009 8 450-456. (doi:10.1021/pr800651j)

32 Vanderpump MP, Tunbridge WM, French JM, Appleton D, Bates D, Clark F, Grimley Evans J, Hasan DM, Rodgers H, Tunbridge F et al. The incidence of thyroid disorders in the community: a twentyyear follow-up of the Whickham Survey. Clinical Endocrinology 199543 55-68. (doi:10.1111/j.1365-2265.1995.tb01894.x)

33 Parekh R, Isenberg D, Rook G, Roitt I, Dwek R \& Rademacher T. A comparative analysis of disease-associated changes in the galactosylation of serum IgG. Journal of Autoimmunity 19892 101-114. (doi:10.1016/0896-8411(89)90148-0)

34 Chen CR, Hamidi S, Braley-Mullen H, Nagayama Y, Bresee C, Aliesky HA, Rapoport B \& McLachlan SM. Antibodies to thyroid peroxidase arise spontaneously with age in NOD.H-2h4 mice and appear after thyroglobulin antibodies. Endocrinology $2010 \mathbf{1 5 1}$ 4583-4593. (doi:10.1210/en.2010-0321)

35 Effraimidis G, Strieder TG, Tijssen JG \& Wiersinga WM. Natural history of the transition from euthyroidism to overt autoimmune hypo- or hyperthyroidism: a prospective study. European Journal of Endocrinology 2011164 107-113. (doi:10.1530/EJE-10-0785)
36 Brent GA \& Davies TF. Hypothyroidism and thyroiditis: classification. In Williams Textbook of Endocrinology, edn 12, Ch. 13, pp 412-439. Eds S Melmed, KS Polonsky, PR Larsen \& HM Kronenberg, Philadelphia, PA: Saunders Elsevier, 2011.

37 Arnold JN, Wormald MR, Sim RB, Rudd PM \& Dwek RA. The impact of glycosylation on the biological function and structure of human immunoglobulins. Annual Review of Immunology 200725 21-50. (doi:10.1146/annurev.immunol.25.022106. 141702)

38 Kinoshita N, Ohno M, Nishiura T, Fujii S, Nishikawa A, Kawakami Y, Uozumi N \& Taniguchi N. Glycosylation at the Fab portion of myeloma immunoglobulin $\mathrm{G}$ and increased fucosylated biantennary sugar chains: structural analysis by high-performance liquid chromatography and antibody-lectin enzyme immunoassay using Lens culinaris agglutinin. Cancer Research $1991515888-5892$.

39 Kiso Y, Furmaniak J, Morteo C \& Smith BR. Analysis of carbohydrate residues on human thyroid peroxidase (TPO) and thyroglobulin $(\mathrm{Tg})$ and effects of deglycosylation, reduction and unfolding on autoantibody binding. Autoimmunity 199212 259-269. (doi:10.3109/08916939209148468)

Received 1 November 2012

Revised version received 17 December 2012

Accepted 29 January 2013 